\title{
Strongly nonexponential time-resolved fluorescence of quantum-dot ensembles in three-dimensional photonic crystals
}

Nikolaev, Ivan S.; Lodahl, Peter; van Driel, A. Floris; Koenderink, A. Femius; Vos, Willem L.

Published in:

Physical Review B Condensed Matter

Link to article, DOI:

10.1103/PhysRevB.75.115302

Publication date:

2007

Document Version

Publisher's PDF, also known as Version of record

Link back to DTU Orbit

Citation (APA):

Nikolaev, I. S., Lodahl, P., van Driel, A. F., Koenderink, A. F., \& Vos, W. L. (2007). Strongly nonexponential timeresolved fluorescence of quantum-dot ensembles in three-dimensional photonic crystals. Physical Review $B$ Condensed Matter, 75(11), 115302. https://doi.org/10.1103/PhysRevB.75.115302

\section{General rights}

Copyright and moral rights for the publications made accessible in the public portal are retained by the authors and/or other copyright owners and it is a condition of accessing publications that users recognise and abide by the legal requirements associated with these rights.

- Users may download and print one copy of any publication from the public portal for the purpose of private study or research.

- You may not further distribute the material or use it for any profit-making activity or commercial gain

- You may freely distribute the URL identifying the publication in the public portal 


\title{
Strongly nonexponential time-resolved fluorescence of quantum-dot ensembles in three-dimensional photonic crystals
}

\author{
Ivan S. Nikolaev, ${ }^{1,2}$ Peter Lodahl, ${ }^{2,3}$ A. Floris van Driel, ${ }^{4}$ A. Femius Koenderink, ${ }^{1}$ and Willem L. Vos ${ }^{1,2, *}$ \\ ${ }^{1}$ FOM Institute for Atomic and Molecular Physics (AMOLF), 407 Amsterdam, The Netherlands \\ ${ }^{2}$ Complex Photonic Systems, MESA ${ }^{+}$Research Institute, University of Twente, 7500 AE Enschede, The Netherlands \\ ${ }^{3}$ COM.DTU, Department of Communications, Optics, and Materials, Nano·DTU, Technical University of Denmark, \\ 2800 Kgs. Lyngby, Denmark \\ ${ }^{4}$ Condensed Matter and Interfaces, Debye Institute, Utrecht University, 3508 TC Utrecht, The Netherlands
}

(Received 14 October 2005; revised manuscript received 17 November 2006; published 1 March 2007)

\begin{abstract}
We observe experimentally that ensembles of quantum dots in three-dimensional (3D) photonic crystals reveal strongly nonexponential time-resolved emission. These complex emission decay curves are analyzed with a continuous distribution of decay rates. The log-normal distribution describes the decays well for all studied lattice parameters. The distribution width is identified with variations of the radiative emission rates of quantum dots with various positions and dipole orientations in the unit cell. We find a striking sixfold change of the width of the distribution by varying the lattice parameter. This interpretation qualitatively agrees with the calculations of the 3D projected local density of states. We therefore conclude that fluorescence decay of ensembles of quantum dots is highly nonexponential to an extent that is controlled by photonic crystals.
\end{abstract}

DOI: 10.1103/PhysRevB.75.115302 PACS number(s): 78.67.Hc, 42.70.Qs, 42.50.Ct, 42.50.Nn

\section{INTRODUCTION}

Control over spontaneous emission from ensembles of excited light sources is of great interest for many applications, such as miniature lasers, light-emitting diodes, ${ }^{1,2}$ and solar cells. ${ }^{3}$ The rate of spontaneous emission is determined not only by the internal nature of emitters but also by their environment. ${ }^{4,5}$ According to Fermi's golden rule, this rate is proportional to the local radiative density of optical states (LDOS), to which the emitters couple. ${ }^{6,7}$ This projected LDOS counts, at given frequency and orientation of the transition dipoles, the number of electromagnetic states at the locations of the emitters. It has been predicted that periodic dielectric structures, known as photonic crystals, can be used to radically change the LDOS. ${ }^{1}$ The main research goal is the achievement of a photonic band gap, i.e., a range of frequencies where no electromagnetic states exist inside the crystal, irrespective of the location. It has also been predicted that a much weaker requirement than a gap suffices to suppress spontaneous emission, ${ }^{6}$ by placing sources at judicious locations in the crystal unit cell where the LDOS vanishes. Since the frequency-integrated number of states is conserved, one expects the LDOS to be strongly increased at some frequencies outside such a pseudogap. ${ }^{6,8-11}$ This means that photonic crystals may completely control the emission rates between complete inhibition and strong enhancement even in the absence of a photonic band gap. Since photonic crystals are a natural platform for solid-state emitters such as quantum dots, ${ }^{12-15}$ such control of spontaneous emission is relevant to applications in quantum information.

Most theoretical papers on spontaneous emission in photonic crystals concern single light sources. ${ }^{6-8,10,11}$ In the case of a weak emitter-field interaction, one expects to see a single-exponential decay curve with a slope equal to the decay rate or inverse lifetime. However, experiments on ensembles of emitters often show nonexponential decays. Such complex decay dynamics can be due to four reasons: (i)
Emitters experience different LDOSs when they are distributed over different positions and dipole orientations in the unit cell of a photonic crystal. (ii) It has been predicted that single sources reveal nonexponential decay due to van Hove singularities in the LDOS. ${ }^{7}$ (iii) Nonexponential decay may appear if the emitters have more internal levels than the usually considered two levels. (iv) Temporal fluctuations of the emitters' environment on time-scales larger than the fluorescence lifetime can lead to apparent nonexponential decays. At any rate, it is an open challenge to interpret complex nonexponential decay curves, in particular, to obtain information on the local density of states.

In this study, we investigate time-resolved spontaneous emission from an ensemble of light sources distributed over a well-defined set of positions in the unit cell. We interpret the emission data with a continuous distribution of emission rates. This distribution is identified with the distribution of the LDOS over all positions $r_{i}$ sampled by sources with fixed emission frequency and random dipole orientations. The distribution width shows a striking sixfold variation with the varying crystal lattice parameter, in qualitative agreement with intricate calculations of the three-dimensional (3D) LDOS. This study opens an avenue to the analysis of timeresolved emission from ensembles of light sources in complex photonic systems.

\section{EXPERIMENTAL DETAILS}

We have studied room-temperature spontaneous emission from sources embedded in fcc inverse opals consisting of air spheres in a titania $\left(\mathrm{TiO}_{2}\right)$ backbone shown in Fig. 1(a). These 3D photonic crystals strongly interact with light. ${ }^{16,17}$ Extensive details on the fabrication and characterization of the inverse opals are reported in Ref. 18. The lattice parameter $a$ is determined from the measured central wavelength of the lowest stop band $\lambda_{c}: a=\sqrt{3} \lambda_{c} /(2 n)$, where $n$ 

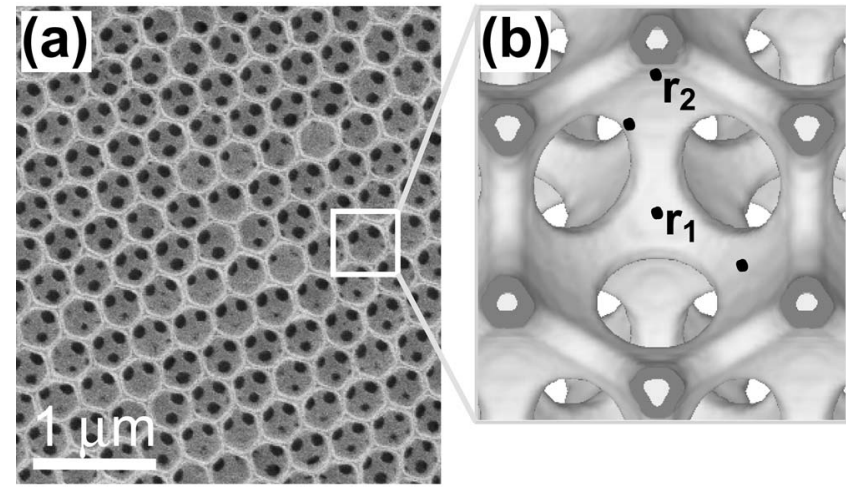

FIG. 1. (a) Electron-microscope image of the (111) surface of an inverse opal consisting of air spheres in $\mathrm{TiO}_{2}$ with lattice parameter $a=425 \mathrm{~nm}$. (b) Schematic of quantum dots (black spots) on the internal titania-air surfaces at symmetry-inequivalent positions $r_{i}$ in the crystal unit cell.

$=1.27 \pm 0.15$ is the average refractive index consistent with 10-20 vol \% $\mathrm{TiO}_{2}$. We have studied 15 different samples with lattice parameters ranging from $a=255 \pm 10 \mathrm{~nm}$ to $a$ $=760 \pm 20 \mathrm{~nm}$.

We use CdSe-ZnSe (core-shell) colloidal quantum dots (QDs) as light sources because of their high fluorescence quantum efficiency and narrow homogeneous spectral width. ${ }^{19}$ The emission spectrum of the QDs is centered at $\lambda=610 \mathrm{~nm}$, which is determined by the average diameter of the nanocrystals of $4.5 \mathrm{~nm}$. A detailed experimental and theoretical study of the emission spectra of QDs inside 3D photonic crystals is presented elsewhere. ${ }^{17}$ The process of the liquid infiltration of the photonic crystals with the QDs is described in Refs. 12 and 17, the QDs precipitate at positions $r_{i}$ that are random but within a well-defined set, on the internal surfaces of the air spheres inside the inverse opals [see Fig. 1(b)], with an estimated low density of four QDs per air sphere.

The QDs are excited at $\lambda=447 \mathrm{~nm}$ with a diode laser (Picoquant) emitting $90 \mathrm{ps}$ pulses with $20 \mathrm{~nJ} /$ pulse. $^{17} \mathrm{We}$ record fluorescence decay curves of QD emission with a microchannel plate photomultiplier tube detector (Hamamatsu R3809U) using the time-correlated single-photon counting method. The decay curves are histograms of the arrival time of a photon emitted after the laser pulse, obtained over many excitation-emission cycles with a resolution better than $100 \mathrm{ps}$. The slope of the decay curves yields a decay rate $\gamma$ $=\gamma_{\text {rad }}+\gamma_{\text {nad }}$, which is the sum of the spontaneous-emission decay rate $\gamma_{\text {rad }}$ and the nonradiative rate $\gamma_{\text {nrad }}$ depopulating the excited states of the QDs.

\section{RESULTS AND DISCUSSION}

In Fig. 2, we show time-resolved spontaneous emission from the QDs in inverse opals with three different lattice parameters $a$, i.e., different reduced frequencies $a / \lambda$. The data were collected at $\lambda=615 \mathrm{~nm}$ within a narrow range $\Delta \lambda=3 \mathrm{~nm}$ to select the same population of QDs with identical emission properties on each sample. For inverse opals with $a=255 \mathrm{~nm}, \lambda=615 \mathrm{~nm}$ is in the low-frequency limit,

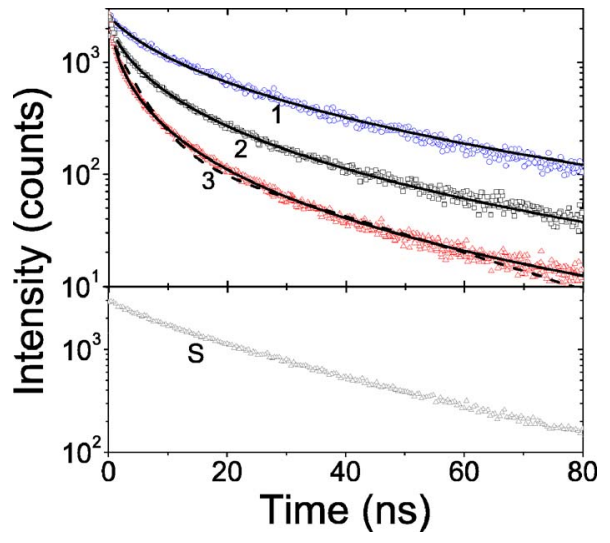

FIG. 2. (Color online) Fluorescence decay curves recorded at $\lambda=615 \mathrm{~nm}$ and $T=295 \mathrm{~K}$ from QDs in inverse opals with lattice parameters of $a=425 \mathrm{~nm}$ (1), $a=255 \mathrm{~nm}$ (2), and $a=540 \mathrm{~nm}$ (3), and from QDs in a different chemical environment, a chloroform suspension (S). The solid lines are fits of the log-normal distribution of decay rates to the data. The goodness of fit $\chi_{\text {red }}^{2}$ varies from 1.1 to 1.4 , close to the ideal value of 1 . The dashed line in (3) shows a heuristic biexponential model, which does not agree with the data $\left(\chi_{\text {red }}^{2}>1.9\right)$.

where the frequency dependence of the LDOS is known to show an $\omega^{2}$ behavior. ${ }^{6,10}$ We see that the spontaneous emission in a sample with $a=425 \mathrm{~nm}$ is inhibited compared to the reference. Conversely, in a sample with $a=540 \mathrm{~nm}$, the decay rate is enhanced. As in any photonic-crystal environment, the backbone also fluoresces, which here distorts the signal at short times. We have carefully removed the $\mathrm{TiO}_{2}$ signal from the measured decay curves since we know its spectrum and decay curve from the measurements on an undoped inverse opal; the backbone has a count rate less than $12 \%$ of the QD signal. We exclude the possibility that QDs at the sample surface contribute any measurable signal: the analysis of separate angle-resolved measurements reveals an excellent agreement with theory for sources emitting in the bulk of photonic crystals. ${ }^{17}$ QDs on the surface are rinsed off after infiltration. Since we also verified that the pump beam was not Bragg diffracted, the pump intensity is maximum inside the samples at a distance of several Bragg attenuation lengths from the surface due to light diffusion. ${ }^{17}$ Therefore, Fig. 2 demonstrates time-resolved emission from an ensemble of QDs controlled by photonic crystals, over a much larger time span than in previous experiments. ${ }^{12-15,20}$

A remarkable feature in Fig. 2 is that the decay curves from the QDs in the inverse opals strongly deviate from a single-exponential decay. To explain this observation, we consider the four reasons discussed above: (i) Since the QDs are distributed over positions and dipole orientations in the unit cell [see Fig. 1(b)], they should indeed experience different LDOSs. (ii) Observation of nonexponential decay due to van Hove singularities in the LDOS requires single-dot experiments, which is not the case here. (iii) Even though the colloidal QDs are not true two-level systems, their emission decay is close to being single exponential, ${ }^{21}$ as confirmed in Fig. 2. (iv) It was suggested in Ref. 22 that temporal fluctuations of the environment surrounding the QDs induce a distribution of nonradiative decay channels. In our experiments, 
however, the nonradiative rates $\gamma_{\text {nrad }}$ hardly vary from sample to sample because QDs from the same batch are used, and the photonic crystals are chemically identical. We observe only minute differences of decay-curve slopes among the samples with the same lattice parameters, which indicates that the temporal fluctuations are identical for all samples. Therefore, we can safely attribute the observed variations of the nonexponential decay curves to a distribution of radiative decay rates $\gamma_{\text {rad }}$ as a result of a spatial and orientational variation of the LDOS.

To interpret the complex, nonexponential decay curves, we propose a different line of attack by modeling the curves with a continuous distribution of decay rates:

$$
I(t)=I(0) \int_{\gamma=0}^{\infty} \phi(\gamma) e^{-\gamma t} d \gamma
$$

where $\phi(\gamma)$ is a distribution of decay rates with dimension of time. The fluorescence intensity $I(t)$ is proportional to the time-derivative of the concentration of excited emitters. Therefore, $\phi(\gamma)$ describes a distribution of the concentration of emitters with a certain $\gamma$, weighted by the corresponding $\gamma_{\text {rad }}{ }^{23}$ This approach has two advantages: first, it enables us to explain intrinsically nonexponential decay curves, and second, the distribution containing physical information on decay rates is readily available, which is essential when treating an ensemble of emitters. We use the log-normal distribution function

$$
\phi(\gamma)=A \exp \left(-\frac{\ln ^{2}\left(\gamma / \gamma_{\mathrm{MF}}\right)}{w^{2}}\right),
$$

where $\gamma_{\mathrm{MF}}$ is the most-frequent decay rate corresponding to the maximum of $\phi(\gamma), w$ is a dimensionless width parameter that determines the distribution width at $1 / e$ :

$$
\Delta \gamma=2 \gamma_{\mathrm{MF}} \sinh w,
$$

and $A$ is the normalization constant, so that $\int_{\gamma=0}^{\infty} \phi(\gamma) d \gamma=1$. The important features of the log-normal distribution are that the logarithmic form of the distribution function excludes unphysical negative decay rates and that it is specified in terms of only two free parameters, $\gamma_{\mathrm{MF}}$ and $\Delta \gamma$. Other multiexponential models are the heuristic biexponential model and the Kohlrausch stretched exponential model that has been employed to QDs outside photonic crystals. ${ }^{22,24}$ Figure 2 shows that the biexponential model does not match our data, even though more free parameters are involved. The stretched exponential model does not match our data either, ${ }^{23}$ which again confirms that the variations we observe are due to LDOS effects in photonic crystals and not to complex emission properties of the QDs. In Fig. 2, it is seen that the log-normal distribution model (solid curves) provides an excellent description of the experimental data.

Figure 3 shows the resulting decay-rate distributions for three lattice parameters. It is remarkable that the log-normal distribution model provides an excellent explanation for all reduced frequencies $a / \lambda$ studied, which below will be seen to agree with calculations. Compared to the low-frequency reference $(a=255 \mathrm{~nm})$, the maximum of the distribution $\phi(\gamma)$ is shifted to lower decay rates for the crystal with $a$

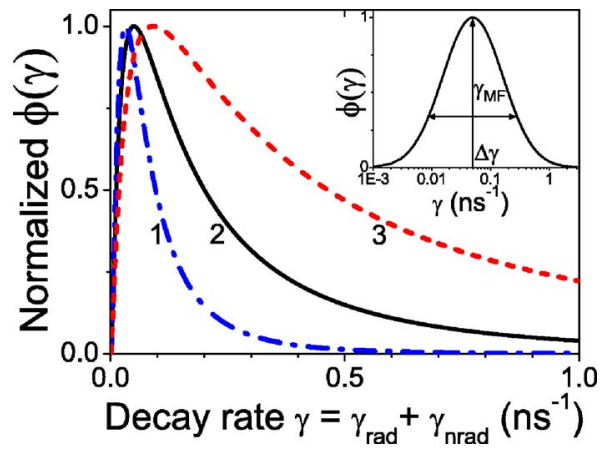

FIG. 3. (Color online) Decay-rate distributions $\phi(\gamma)$ for the inverse opals with lattice parameters $a=425 \mathrm{~nm}(1), a=255 \mathrm{~nm}(2)$, and $a=540 \mathrm{~nm}$ (3), corresponding to the data shown in Fig. 2. The inset shows $\phi(\gamma)$ for $a=255 \mathrm{~nm}$ in the semilogarithmic scale. Clear modifications of $\Delta \gamma$ and $\gamma_{\mathrm{MF}}$ with varying lattice parameter of the inverse opals are seen.

$=425 \mathrm{~nm}$ and to higher rates for the crystal with $a$ $=540 \mathrm{~nm}$. These shifts are a clear demonstration of a photonic effect of the inverse opals on the ensemble of embedded emitters. In Fig. 3, we see a dramatic change of the width $\Delta \gamma$ of the distribution. The large width of each distribution is identified with the variation of the radiative emission rates due to orientational and positional $r_{i}$ dependences of the LDOS at each lattice parameter. Consequently, the decay rates of individual QDs are much more strongly modified by the photonic crystal than the most-frequent rate $\gamma_{\mathrm{MF}}$ of the ensemble.

In Figs. 4(a) and 4(b), we have plotted the resulting values for $\Delta \gamma$ and $\gamma_{\mathrm{MF}}$ vs the reduced frequency. Let us briefly consider $\gamma_{\mathrm{MF}}$ : because the nonradiative part of the total decay rates does not change with the lattice parameter, the change of $\gamma_{\mathrm{MF}}-\gamma_{\mathrm{MF}}^{e f}$ is purely radiative and related to an averaged photonic-crystal LDOS. We compare the experimental data to the calculated density of states (DOS) - the unit-cell average of the LDOS (dashed curve). The measured variation of $\gamma_{\mathrm{MF}}$ is seemingly in good agreement with the DOS. Both inhibited and enhanced decay rates are observed, and the experimental variation in $\gamma_{\mathrm{MF}}$ amounts to a factor of 3. In contrast, the width $\Delta \gamma$ shows a striking sixfold variation, much larger than the change of $\gamma_{\mathrm{MF}}$ [Fig. 4(a)]. Already in the low-frequency limit, $a / \lambda=0.4$, there is a spatial variation of the radiative rate $(\Delta \gamma \geqslant 0)$ because the QDs distributed over inequivalent positions in the unit cell couple to different electric fields. ${ }^{25}$ At the frequencies of the $L$-gap, $a / \lambda \approx 0.7$, the radiative rate $\gamma_{\text {rad }}$ is inhibited in most places in the unit cell that are occupied by the QDs, as confirmed by a low continuous-wave (cw) count rate of only $\approx 2.5 \mathrm{kHz}$. Therefore, the observed narrow width $\Delta \gamma=0.1 \mathrm{~ns}^{-1}$ is a measure of the distribution width of nonradiative rates. In contrast, at $a / \lambda=0.88, \Delta \gamma$ is strongly increased; here, the cw count rate is $\approx 56 \mathrm{kHz}$ at similar experimental conditions, in agreement with an enhanced $\gamma_{\text {rad }}$. We therefore conclude that the large widths $\Delta \gamma$ are determined by a broad distribution of radiative emission rates $\Delta \gamma_{\text {rad }}$ that are proportional to a broad distribution of the projected LDOS at fixed frequency. Hence, the width $\Delta \gamma$ is a much more characteristic parameter to describe the ensemble emission in $3 \mathrm{D}$ photonic crystals than the usually used average rates. ${ }^{12}$ 


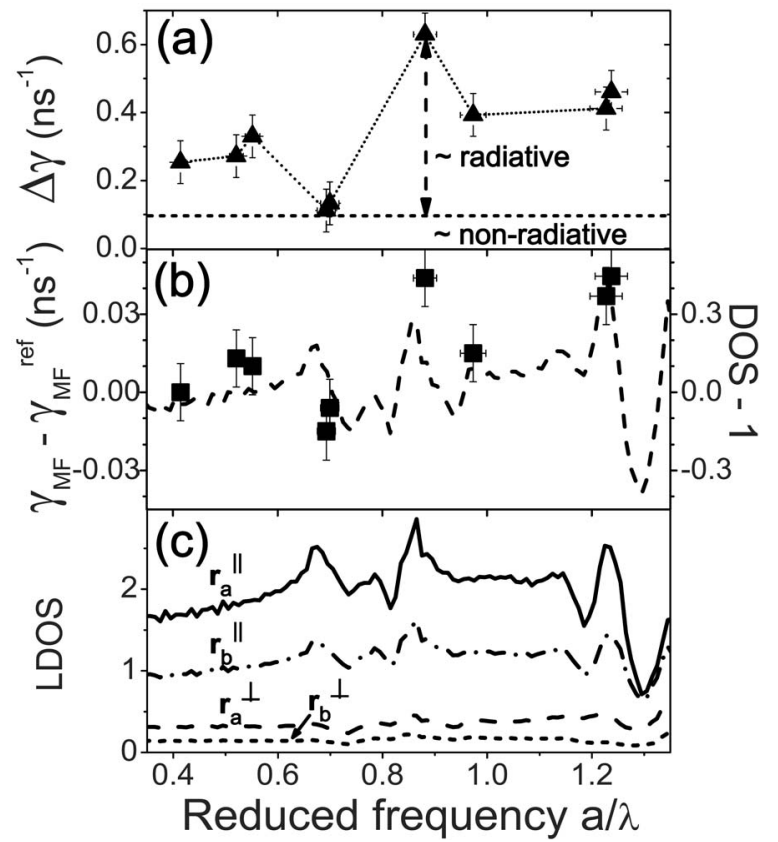

FIG. 4. (a) Width of the decay-rate distribution $\Delta \gamma$ vs lattice parameter at a fixed emission wavelength (triangles) (the dashed curve is a guide to the eye). (b) Difference between $\gamma_{\mathrm{MF}}$ measured on a photonic sample and $\gamma_{\mathrm{MF}}^{r e f}=0.05 \mathrm{~ns}^{-1}$ of the low-frequency reference (squares). The error bars are estimated from the largest difference between data on the samples with similar $a$. The dashed curve represents the relative DOS in the inverse opals. The relative (L)DOS in the inverse opals is the (L)DOS divided by DOS in a homogeneous medium with the same average refractive index ( $n$ =1.27). (c) Relative LDOS at two positions on the internal $\mathrm{TiO}_{2}$-air surfaces projected on dipole orientations parallel $(\|)$ or normal $(\perp)$ to the internal surface: at $r_{a}=0.144(1,1,2)$ and $r_{b}=0.2(1,1,1)$.

We have managed to perform intensive computations of the 3D LDOS at two representative positions in the unit cell at the $\mathrm{TiO}_{2}$-air interface [see Fig. 1(b)]. The LDOS shown in Fig. 4(c) was calculated for dipole orientations parallel and perpendicular to the $\mathrm{TiO}_{2}$-air interface. The calculations were performed using 725 reciprocal-lattice vectors in the $H$-field plane-wave expansion method. ${ }^{10,11}$ The inverse opals were modeled as close-packed air spheres surrounded by shells of $\mathrm{TiO}_{2}(\epsilon=6.5)$ with cylindrical windows between neighboring spheres. The model agrees with prior optical experiments. ${ }^{26}$ The integration over wave vectors $\mathbf{k}$ was performed by representing the full Brillouin zone by an equidistant k-point grid consisting of 291416 points. ${ }^{11}$ The results in Fig. 4(c) reveal a strong dependence of the LDOS both on the position in the crystal unit cell (compare curves 1 and 2) and on the dipole orientation (compare curves 1 and 3). It is remarkable that in the relevant $a / \lambda$ range, the dependence of the LDOS on the lattice parameter is the same at both positions $r_{i}$ and both orientations, and even as the unit-cell averaged LDOS [Fig. 4(b)]. This result agrees with the observation that all measured decay curves are successfully modeled with the same log-normal shape of the decay-rate distribution. Because the LDOS for dipoles perpendicular to the interface is inhibited and nearly constant at all reduced frequencies, whereas the LDOS for parallel dipoles strongly varies, we propose that the width of the LDOS distribution has a similar frequency dependence as the LDOS itself. This notion agrees with our observation that $\Delta \gamma$ tracks the behavior of $\gamma_{\mathrm{MF}}$. A quantitative comparison of our data to the calculated LDOS is a challenge, since detailed knowledge is needed on the relation between $\gamma_{\text {rad }}$ and $\gamma$ to infer the true radiative decay-rate distribution (see Ref. 23). Qualitatively, the calculated LDOS reflects the main features of our experiments.

\section{SUMMARY}

We have successfully explained highly nonexponential decay curves of an ensemble of QDs in 3D photonic crystals with a continuous distribution of decay rates. We relate this distribution to the fact that QDs in various positions in the unit cell with random dipole orientations experience different LDOSs. It is gratifying that recent calculations for Bragg onion resonators also consider nonexponential decay for similar reasons. ${ }^{27}$

Our theoretical results demonstrate that large inhibitions and enhancements of the spontaneous emission can be achieved with properly positioned and oriented efficient dipolar light sources inside 3D photonic crystals, at room temperatures and in large volumes limited only by the crystal size. The complementary case of a single QD in an opal has recently been studied in Ref. 28, and for a single oriented quantum dot in a two-dimensional slab, interesting steps have been discussed in Refs. 13, 15, and 29.

\section{ACKNOWLEDGMENTS}

We thank Léon Woldering for making the inverse opals, Karin Overgaag for experimental help, and Ad Lagendijk and Daniël Vanmaekelbergh for stimulating discussions. This work is a part of the research program of the Stichting voor Fundamenteel Onderzoek der Materie (FOM) and of Chemische Wetenschappen $(\mathrm{CW})$ that are financially supported by the Nederlandse Organisatie voor Wetenschappelijk Onderzoek (NWO).
*Electronic address: w.1.vos@amolf.nl. URL: www.photonicbandgaps.com

${ }^{1}$ E. Yablonovitch, Phys. Rev. Lett. 58, 2059 (1987).

${ }^{2}$ H.-G. Park, S.-H. Kim, S.-H. Kwon, Y.-G. Ju, J.-K. Yang, J.-H. Baek, S.-B. Kim, and Y.-H. Lee, Science 305, 1444 (2004).
${ }^{3}$ M. Grätzel, Nature (London) 414, 338 (2001).

${ }^{4}$ K. H. Drexhage, J. Lumin. 12, 693 (1970).

${ }^{5}$ D. Kleppner, Phys. Rev. Lett. 47, 233 (1981).

${ }^{6}$ R. Sprik, B. A. van Tiggelen, and A. Lagendijk, Europhys. Lett. 35, 265 (1996). 
${ }^{7}$ N. Vats, S. John, and K. Busch, Phys. Rev. A 65, 043808 (2002). ${ }^{8}$ T. Suzuki and P. K. L. Yu, J. Opt. Soc. Am. B 12, 570 (1995).

${ }^{9}$ S. M. Barnett and R. Loudon, Phys. Rev. Lett. 77, 2444 (1996).

${ }^{10}$ K. Busch and S. John, Phys. Rev. E 58, 3896 (1998).

${ }^{11}$ R. Wang, X.-H. Wang, B.-Y. Gu, and G.-Z. Yang, Phys. Rev. B 67, 155114 (2003).

${ }^{12}$ P. Lodahl, A. F. van Driel, I. S. Nikolaev, A. Irman, K. Overgaag, D. Vanmaekelbergh, and W. L. Vos, Nature (London) 430, 654 (2004).

${ }^{13}$ A. Badolato, K. Hennessy, M. Atature, J. Dreiser, E. Hu, P. M. Petroff, and A. Imamoglu, Science 308, 1158 (2005).

${ }^{14}$ A. Kress, F. Hofbauer, N. Reinelt, M. Kaniber, H. J. Krenner, R. Meyer, G. Böhm, and J. J. Finley, Phys. Rev. B 71, 241304 (2005).

${ }^{15}$ D. Englund, D. Fattal, E. Waks, G. Solomon, B. Zhang, T. Nakaoka, Y. Arakawa, Y. Yamamoto, and J. Vučković, Phys. Rev. Lett. 95, 013904 (2005).

${ }^{16}$ A. F. Koenderink, L. Bechger, H. P. Schriemer, A. Lagendijk, and W. L. Vos, Phys. Rev. Lett. 88, 143903 (2002).

${ }^{17}$ I. S. Nikolaev, P. Lodahl, and W. L. Vos, Phys. Rev. A 71, 053813 (2005).

${ }^{18}$ J. E. G. J. Wijnhoven, L. Bechger, and W. L. Vos, Chem. Mater. 13, 4486 (2001).
${ }^{19}$ B. O. Dabbousi, J. Rodriguez-Viejo, F. V. Mikulec, J. R. Heine, H. Mattoussi, R. Ober, K. F. Jensen, and M. G. Bawendi, J. Phys. Chem. B 101, 9463 (1997).

${ }^{20}$ M. Fujita, S. Takahashi, Y. Tanaka, T. Asano, and S. Noda, Science 308, 1296 (2005).

${ }^{21}$ A. F. van Driel, G. Allan, C. Delerue, P. Lodahl, W. L. Vos, and D. Vanmaekelbergh, Phys. Rev. Lett. 95, 236804 (2005).

${ }^{22}$ G. Schlegel, J. Bohnenberger, I. Potapova, and A. Mews, Phys. Rev. Lett. 88, 137401 (2002); B. R. Fisher, H.-J. Eisler, N. E. Stott, and M. G. Bawendi, J. Phys. Chem. B 108, 143 (2004).

${ }^{23}$ A. F. van Driel, I. S. Nikolaev, P. Vergeer, P. Lodahl, D. Vanmaekelbergh, and W. L. Vos, Phys. Rev. B 75, 035329 (2007).

${ }^{24}$ J. Kalkman, H. Gersen, L. Kuipers, and A. Polman, Phys. Rev. B 73, 075317 (2006).

${ }^{25}$ H. Miyazaki and K. Ohtaka, Phys. Rev. B 58, 6920 (1998).

${ }^{26}$ W. L. Vos and H. M. van Driel, Phys. Lett. A 272, 101 (2000).

${ }^{27}$ W. Liang, Y. Huang, A. Yariv, Y. Xu, and S. Y. Lin, Opt. Express 14, 7398 (2006).

${ }^{28}$ M. Barth, R. Schuster, A. Gruber, and F. Cichos, Phys. Rev. Lett. 96, 243902 (2006).

${ }^{29}$ A. F. Koenderink, M. Kafesaki, C. M. Soukoulis, and V. Sandoghdar, Opt. Lett. 30, 3210 (2005). 